\title{
CLINICAL AND LABORATORY PROTOCOL IN SUSPECTED CASES OF BREAST IMPLANT- ASSOCIATED ANAPLASTIC LYMPHOMA
}

Luciana da Fonseca Santos¹, Idam de Oliveira Junior ${ }^{1}$, Luciano Neder², Wilson Eduardo Furlan Matos Alves', Rene Aloisio da Costa Vieira ${ }^{3}$

${ }^{1}$ Hospital de Câncer de Barretos - Barretos (SP), Brazil.

${ }^{2}$ Faculdade de Medicina de Ribeirão Preto - Ribeirão Preto (SP), Brazil.

${ }^{3}$ Hospital de Câncer de Muriaé - Muriaé (MG), Brazil.

Introduction: Breast implant-associated anaplastic large cell lymphoma (BIA-ALCL) is a rare entity. As a result, care in the diagnostic flowchart is not systematized and known by all mastologists. Non-systematic evaluation can lead to falsenegative assessments, making it crucial to have systematic knowledge about the subject. Methods: The Research Ethics Committee of the Hospital de Câncer de Barretos approved this study, under the number 23026719.5.0000.5437/2019. We sought to conduct an integrative review on the subject, aiming at identifying the diagnostic flowchart and therapeutic care to be adopted by mastologists. Results: BIA-ALCL diagnosis should be considered in patients with peri-implant seroma when the implant was placed for more than a year. Despite being more associated with textured implants, the disease was also identified in smooth implants. In clinical practice, breast ultrasound is the examination of choice. The main finding is peri-implant seroma, with puncture. Breast magnetic resonance imaging can help evaluate implant integrity. Positron emission tomography-computed tomography (PET-CT) can be used in high suspicion or confirmed cases, improving the staging. Some precautions are essential in the care of the collected fluid; otherwise, it can lead to false negatives. Cytology should be performed on the day of collection, in less than 6 hours. Centrifuging the fluid collected should be considered in order to obtain a smaller volume but with high cellularity. The centrifuged material can be processed to form a paraffin block (cell block), in which the Wright-Giemsa stain will be used. The material can be sent immediately or after adding $50 \%$ alcohol. Surgery should be indicated in cases of tumor mass, high clinical suspicion, or recurrent seroma. In these cases, drainage of the peri-implant content and capsulectomy with concomitant removal of the breast implant should be carried out. Bilateral implant removal is recommended if BIA-ALCL is confirmed; however, in case of suspected diagnosis, the indication becomes questionable, and the surgeon must discuss the situation with the patient. Conclusion: Diagnostic systematization reduces the possibility of false-negative results, improving the care described above. 